\title{
Interfacing with Robots without the use of Touch or Speech
}

\author{
Addison Clark \\ addison.clark@mavs.uta.edu \\ The University of Texas at Arlington \\ Arlington, Texas, USA
}

\author{
Ishfaq Ahmad \\ iahmad@cse.uta.edu \\ The University of Texas at Arlington \\ Arlington, Texas, USA
}

\begin{abstract}
As the field of robotics develops, so do the methods of interfacing with and controlling those robots. Many modern robots can be communicated with using commands that require neither speech nor touch. The two main motivations behind this trend are the assistance of people with disabilities and the desire for more natural Human-Robot Interaction (HRI). In allowing nonverbal communication with robots, accessibility of the systems increases to allow more people to interact with and benefit from robotics systems. Additionally, nonverbal communication provides more natural communication, which can lead to many benefits in HRI. This paper provides an overview of existing technologies in touchless and nonverbal human-robot interfaces; it presents the most prevalent interface methods, their uses, and limitations. Discussions are provided on future directions for research.
\end{abstract}

\section{CCS CONCEPTS}

- Social and professional topics $\rightarrow$ People with disabilities; • Hardware $\rightarrow$ Neural systems; Emerging interfaces; • Human-centered computing $\rightarrow$ Accessibility; • Computer systems organization $\rightarrow$ Robotics.

\section{KEYWORDS}

HRI, gaze detection, EEG, emotion detection

ACM Reference Format:

Addison Clark and Ishfaq Ahmad. 2021. Interfacing with Robots without the use of Touch or Speech. In The 14th PErvasive Technologies Related to Assistive Environments Conference (PETRA 2021), fune 29-7uly 2, 2021, Corfu, Greece. ACM, New York, NY, USA, 7 pages. https://doi.org/10.1145/3453892.3461330

\section{INTRODUCTION}

As the field of robotics has developed, interface methods have expanded to include a multitude of different controls. Many robots in recent years have been created to understand commands that require neither touch nor speech. Motivations behind this trend include assisting people with disabilities $(\mathrm{PwD})$ and providing more natural Human-Robot Interaction (HRI) to people in general. Assistive robots can provide physical, therapeutic, and social services to people with disabilities [12]. People with certain severe disabilities

Permission to make digital or hard copies of all or part of this work for personal or classroom use is granted without fee provided that copies are not made or distributed for profit or commercial advantage and that copies bear this notice and the full citation on the first page. Copyrights for components of this work owned by others than ACM must be honored. Abstracting with credit is permitted. To copy otherwise, or republish, to post on servers or to redistribute to lists, requires prior specific permission and/or a fee. Request permissions from permissions@acm.org.

PETRA 2021, June 29-fuly 2, 2021, Corfu, Greece

(C) 2021 Association for Computing Machinery.

ACM ISBN 978-1-4503-8792-7/21/06 . \$ \$15.00

https://doi.org/10.1145/3453892.3461330 cannot interact with these robots in standard ways; for example, an individual with upper body paralysis would be unable to press buttons. The robot could still be commanded with other methods, however, such as eye-tracking. Disability studies is a rapidly growing field; as society continues to increase understanding of PwD, assistive technology will have many directions to grow and develop in order to support them.

More natural HRI is also a prevalent factor in the development of touchless and nonverbal robot interfaces. Nonverbal communication accounts for $65 \%$ of human communication, including gestures, facial expressions, and gaze [28]. As robots advance, users will expect them to act more like humans. More natural interaction will also encourage people to use robots more, allowing further assistance, entertainment, and productivity. As nonverbal communication with robots becomes more advanced, robots are likely to become much more common in everyday use. HRI includes the study of how robots should react given specific stimuli and how those reactions can be tailored to elicit specific responses in the human users. By developing touchless and nonverbal interfaces, robots can receive many more inputs from a given conversation, similar to typical human interaction. Thus, HRI can become more advanced as robots are able to react to more stimuli, giving an increasingly more natural and personal response.

There are many research challenges in the field of touchless and nonverbal interfaces. One is a lack of understanding in some parts of human communication. There is research in the role of gestures and eye contact in communication, such as [7, 13, 23], but there is still much that should be applied to robotics. The ways in which nonverbal communication effects different listeners cognitively and emotionally is not wholly understood. To apply some of these concepts to robotics would require an understanding of what programmed behavior would be likely to elicit a specific response from the user.

Another important challenge lies in the devices used to collect and decode touchless and nonverbal information. Eye tracking devices have limitations in use, such as the user remaining stagnant in a limited field of view. Computer vision could be a more versatile method of eye tracking, but that has its own challenges. Computer vision is dependent on lighting and background elements in a way that infrared eye trackers are not. Although it uses only a camera, which are built into many robots and could follow the user as they move, it requires more processing and methods that are not yet consistent in uncontrolled environments. Gesture recognition also often uses computer vision and thus suffers from the same problems.

Electroencephalogram (EEG) has many of the same problems but often to an even further degree. The understanding of the brain is much less than that of eye contact and gestures. EEG devices can receive electrical signals from the brain as input, but the meaning of 


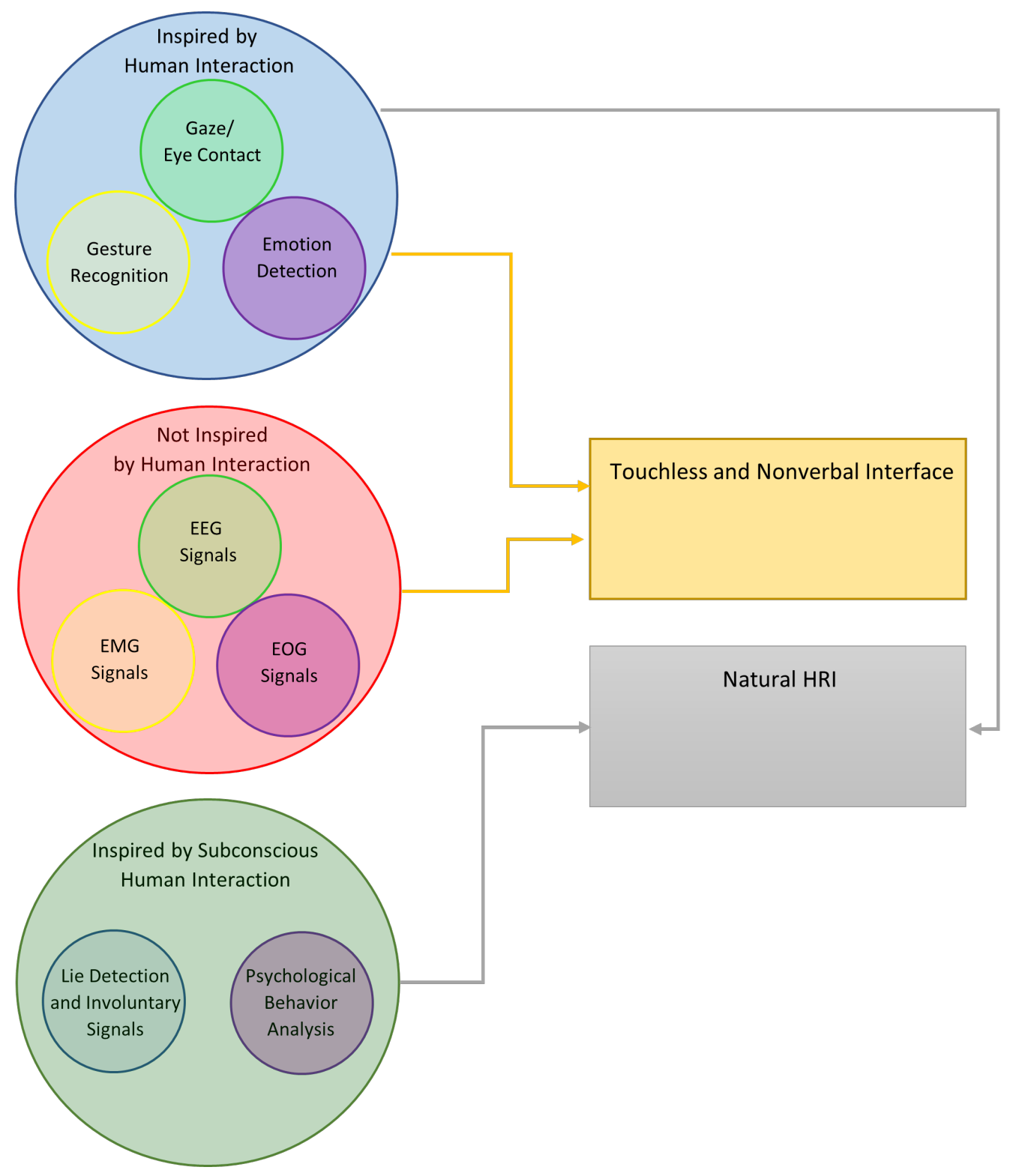

Figure 1: Classification of touchless and nonverbal systems and the functions of which they are composed

specific signals is often unknown. Several types of commonly used signals are discussed later in Section 3, but many generalized brain signals are not understood and therefore cannot often be used as commands in an interface. EEG devices themselves have significant limitations as well. One such limitation is a low signal to noise ratio (SNR). EEG filtering is often a considerable challenge due to this fact. Electrode helmets can also be cumbersome and reliable for only a short time; some helmets require electrodes to be wetted to properly conduct signals, which means they can only operate for a limited period before the electrodes dry and the signal strength is reduced. Other limitations are discussed in depth later in the paper.

Touchless and nonverbal interfaces include all communication between a human and a robot that includes neither touch nor speech.
Common examples of these interfaces are gaze detection, EEG or brainwave communication, gestures, and emotion detection. Each of these methods has different benefits and varied amounts of study. With the exception of EEG communication, all are inspired by typical human communication. The layout of the paper is as follows: Section 2 provides an overview of gaze-based robot interfaces and their applications and challenges; Section 3 outlines the same for EEG-based interfaces. Section 4 discusses other nonverbal signals, including emotion recognition, gestures, and lie detection. Section 5 provides some conclusions and future work. 


\section{GAZE BASED ROBOT INTERFACES}

Gaze is one of the most important nonverbal cues in human interactions. It has also been used as a control mechanism for people with disabilities for many years [3]. Gaze tracking methods include pupil detection and head angle detection using computer vision, infrared projection eye tracking, and flex sensors. Computer vision methods are developed using cameras, either in the robots or external. Infrared projection eye tracking includes devices like Tobii and Eye Tribe eye trackers and can be used when the user remains relatively static in front of the device. Flex sensors were used in tandem with vision to increase accuracy. The following section will explore the above methods of gaze tracking and their uses on robot interfaces.

\section{Table 1: Methods of Gaze Detection for Robot Interfaces}

\begin{tabular}{|l|l|}
\hline Method & References \\
\hline Near-Infrared Eye Trackers & {$[9,11,14,30]$} \\
\hline Vision-Based Eye Detection & {$[19]$} \\
\hline Head-Mounted Eye Trackers & {$[27]$} \\
\hline Sensors Combined with Vision & {$[2]$} \\
\hline
\end{tabular}

\subsection{Near-Infrared Eye Trackers}

Near-infrared eye trackers are the most commonly used method for gaze-based robot systems as of the writing of this paper. They are relatively easy to set up and provide accurate results, some trackers providing accuracy within $1^{\circ}$ [1]. These systems require the user to be within a specific volume of space in order to provide accurate results. Given this, they can be useful for control of telepresence or surveillance robots in which the user remains seated or does not have to perform other tasks.

One study used the Eyelink 1000 Plus eye tracker to control a robot arm [11]. The arm was positioned in front of a board with a grid of numbers. The robot had a marker attached to the end and a paper mask face. The goal of the participants was to mark a number on the grid using the robot. Given only the information that the robot could be controlled using gaze, all participants were able to find and use one or more of the control strategies. Commands were executed rather slowly because of the duration of gaze required at the robot's face and the desired number. This would be useful, however, for environments with many distractions to avoid unwanted controls due to quick attention shifts.

In [9], the Gazepoint GP3 eye tracker was used command a humanoid robot via simple shapes drawn with eye movements. The user was presented with a screen showing the view from the humanoid robot NAO and an overlay of commands. At any time, only three commands could be given as the system recognizes three shapes. Given a tennis ball, the user could draw a circle to rotate the ball, a square to kick the ball, or a triangle to pick up the ball. Each command was executed using NAO's integrated software. Shape based command recognition was comparable between gaze-drawn and mouse-drawn shapes.

Telepresence robots can also be controlled using infrared eye trackers. In a 2019 study, a virtual reality headset was used to control a telepresence robot, using both a controller (Xbox 360 controller) and eye tracking native to the headset [30]. Gaze controls were found to be slower and less accurate than the traditional controller. The researchers have planned a further study to observe the effects of first training the participants on the controls so that they are not unfamiliar during the trials.

Gaze-based interfaces have similar applications in control of Unmanned Aerial Vehicles (UAVs), or drones. In [14], four different control types are tested using a combination of gaze data collected from an Eye Tribe gaze tracker and keyboard. Four different controls are identified: speed (forward/ backward movement), rotation (around the z-axis), translation (left/ right movement), and altitude. From these four, either translation or rotation were mapped to $\mathrm{x}$-axis gaze control while either speed or altitude were mapped to $\mathrm{y}$ axis gaze control. These made the four different control types, with two mapped to the axes of gaze tracking and the other two mapped to keyboard in all combinations. The participants stated a preference for using gaze to control rotation ( $\mathrm{x}$-axis of the eye tracker) and speed (y-axis of eye tracker) while the keyboard-controlled altitude and translation.

\subsection{Computer Vision Based Command Recognition}

Computer vision is arguably a much more versatile method of gaze tracking on robots. Many robots are already equipped with a camera that can be used for vision. Vision would also allow the user to move around while interacting with or controlling the robot, unlike the aforementioned infrared based eye trackers. Despite these facts, vision appears to be much less commonly used for human-robot interfaces.

In [19] two humanoid robots are used to react to human gaze in order to play a simple, gaze-based game. Two robots and two humans were set up to play the Wink Murder party game, in which a random participant is assigned the role of murderer and must wink at other participants to kill them. However, if an innocent is looking at the murderer when they kill another participant, the murderer is caught and the innocents win. In this paper, the robots would detect the gaze of the humans using eye-corner detection for head pose tracking and iris center detection for gaze direction tracking.

The gaze detection was used to determine if the robot should kill the participant it was looking at or if it was being observed. If the robot were an innocent, it would detect the kill action only on a human that it was directly looking at. The robots were able to detect gaze accurately enough to be on par with the humans in the game. However, this experiment required an external camera, as the in-eye cameras on the robots were too low resolution to accurately detect gaze. In the future, a similar game could be implemented on robots with higher resolution mounted cameras to see if the robots could still perform adequately without having an external camera allowing them a wider field of vision than humans.

\subsection{Other Gaze Detection Methods}

Another method of gaze detection is head mounted eye trackers. Head mounted eye tracking devices are similar to near-infrared eye trackers but are mounted on the head to allow for more dynamic 
environments. Such devices remove some of the limitations of traditional eye trackers and would allow many different scenarios of HRI and gaze interfaces. In [27], a head mounted eye tracker is used to determine human responses to robot gaze action. Eye contact and face gazes are known to engage humans during human-human interactions $[7,13]$. This study explored if the same is true for HRI. The experiment involved a humanoid robot (NAO) following the gaze of the participant, either making eye contact or looking at an object. They found that the participants were very responsive to the face looks of the robot and that more face looks elicited by the robot have the potential to strengthen the social influence and the user's social engagement.

There are other methods of gaze detection that have not been widely integrated into human-robot interfaces. One such method is the addition of flex sensors on the neck to vision, allowing more accurate results on rotated faces, such as in [2]. Though the flex sensors may prove intrusive for everyday use, they could be helpful for studying effective gaze-based interfaces

\section{EEG BASED ROBOT INTERFACES}

Electroencephalogram (EEG) is a method of recording brain activity in which electrodes are placed in specific locations on the head. Many consumer level EEG helmets exist, with varying numbers of electrodes, amount of required setup, cost, accuracy, and SNR. These helmets communicate with computers and are used in Brain Computer Interfaces (BCI). The following section will explore human robot interfaces using EEG, as well as methods of increasing EEG signal and classification accuracy to allow for better system control.

Table 2: EEG Signal Types

\begin{tabular}{|l|l|}
\hline Signal Type & References \\
\hline SSVEP & {$[26,31]$} \\
\hline P300 & {$[4,17]$} \\
\hline Motor Imagery & {$[24]$} \\
\hline Feature Extraction and Classification & {$[6,29]$} \\
\hline Other & {$[18]$ (not specified) } \\
\hline
\end{tabular}

\subsection{EEG Only Interfaces}

There are many kinds of brain signals that can be collected from a standard EEG helmet. One such signal is steady-state visual evoked potential (SSVEP), in which specific frequencies naturally evoke a response in the human brain when seen. SSVEP can be used by providing control blocks that flicker at a certain frequency. When the user looks at that area, the EEG helmet will pick up a specific signal which can trigger a command, such as in [31]. Here, a robot's movement is controlled via SSVEP. The interface for the SSVEP was displayed over the camera of the robot, such that each region of the robot's vision displayed a different number flashing at a different rate. In this way, the subject could look at a region for the robot to move to and it would move there, as verse as having separate controls for forward, left, right, etc. The majority (9/12) of the test runs were successful.
EEG signals can also be used directly, with machine learning classifiers translating them into specific commands. In [29], the 14 channel Emotive Epoc+ EEG Headset is used to command a custom humanoid robot. The user concentrates on actions, in this case lean left, lean right, and squat, which are classified using the Emotiv Control Panel. The robot is able to perform the three actions with an accuracy of $70 \%$. However, the system is limited by a significant delay that would disallow its use in real-time scenarios. It is also limited by the EEG technology used. The Emotive Epoch+ uses saline wetted electrodes; these dry after about an hour, meaning that the system can only be used for an hour before the signal and accuracy degrade.

A 2016 study used SSVEP to control a robotic car and the camera on the car [26]. A custom graphical user interface was created to display the video feed from the robot's camera as well as four buttons. The user could select "drive forward," "turn left," "turn right," and "switch to camera mode" while in drive mode. Camera mode had four similar options but for camera control. "Drive forward" was an autonomous program on the robot that followed lines on the ground, and thus depended on the specific test setup. On 61 subjects, the system achieved a mean accuracy of $93 \%$, measured as correct commands divided by total commands given for each subject. This system depends on a user being seated in front of a screen and a very limited number of control options, including a "drive forward" command that depends on a specific environment setup.

Another EEG command method is P300. The P300 wave is an event-related potential (ERP) component involved in the decisionmaking process. It marks a person's reaction to a stimulus, and thus can be used to determine a user's selection. In [4], P300 is used to control a humanoid robot. The user views four to six images of objects from the robot's camera and concentrates on one object. The image borders then flash; when the image that the user is concentrating on flashes, the P300 wave is generated in their brain. The robot is then sent the command to move to and pick up the corresponding object. At four objects, an accuracy of $95 \%$ was achieved with one command taking five seconds due to the number of flashes of the image.

P300 and SSVEP can provide high accuracy and control complicated systems and behaviors. However, both rely on a screen to display flashes. The user therefore often must remain stationary and in front of a screen. While this method is useful for remote controlled robots, it is less useful for things like wheelchair control or HRI where the user would be observing the world themselves, and not through the robot; this is similar to the limitations of near-infrared eye trackers. Future studies could include augmented reality (AR) or a setup with LEDs on objects to allow flashing images without the requirement of the user remaining stationary. However, these methods can also give users vision fatigue from staring at a screen and flashing lights for extended periods of time. Despite the high accuracy and relatively large number of possible commands, SSVEP and P300 are not suitable for many real-world tasks or for extended use by many users who may have sensitive vision.

Generic classification of EEG signals has fewer requirements, but also typically achieves much lower classification accuracy, as seen in [29]. Increasing this accuracy would allow the EEG control 
of robots in completely natural, unaltered environments, and in real-time situations.

\subsection{EEG Combined with Other Methods}

In [17], electrooculography (EOG) is used to make up for the processing time limitations of P300. EOG detects eye movements, such as gaze directions, winks, blinks, and frowns. It is also much faster than ERPs. In this experiment, EOG was used for quick commands, such as movement and switching modes, while EEG was used for other functions, such as picking up objects or selecting one of several robots. The system was able to produce high levels of accuracy for both ERPs and all EOG commands. Subjects were mostly able to adjust to the interface and perform the correct commands. Some subjects were able to perform the tasks in times comparable to hand controls used for the same tasks.

Another non-invasive biological signal that can be combined with EEG is electromyogram (EMG), the measurement of electrical signals from the muscles, typically in the forearm. EMG can be used to detect gestures and hand movements. In [6], a feature extraction technique for EEG-EMG is proposed and compared to the traditional EEG only technique. The combination of the two signals is able to produce higher accuracy than EEG only, and thus could be useful for many applications. It is tested on a robotic hand exoskeleton, which is able to accurately provide feedback to users.

EMG itself also often has accuracy problems; it must be placed in the exact location on the forearm each time it is used. Otherwise the collected data will vary significantly from the training data and the system will have to be retrained for each use. EMG also suffers from a low SNR, similar to EEG. The combination of the two methods can help increase the accuracy. However, EMG interfaces are difficult to implement in real-world devices due to the problem of device placement.

EEG and EOG are combined in [24] to control a hand exoskeleton for people with quadriplegia. In this study, motor imagery is used to control the grasping of the exoskeleton. The user imagines grasping their hand, and the EEG signal is translated into a grasping action by the robot. The device allowed users independent actions of daily living that they were previously unable to perform. EOG was used as a quick cancellation command.

[18] makes use of EEG, EMG, and EOG to control a robotic arm. The robot arm has 5 degrees of freedom, making it significantly more complicated than the exoskeleton in [24]. For this reason, the system makes use of more command types. Given the limitations of EEG, EMG, and EOG alone, all three are used to allow for multiple commands while still maintaining high classification accuracy for each individual signal type. Several different modes were tested, with different combinations of EMG, EOG, and head movement signals used for different movement and grasping commands. The study does not make it clear what type of EEG signal or classification was used for arm control. There are several limitations to the system presented in [18]; given that the primary signals used are EOG and jaw EMG, the user is likely to become fatigued over long periods of use. The system was only tested on one subject, who was involved in the development of the interface. Assistive devices should be tested with the PwD populations that they are intended for to attain useful information on the usability of the device. However, there are ideas presented in [18] that could be expanded upon further and tested with a relevant test group.

\section{OTHER NONVERBAL SIGNALS}

\subsection{Emotion Recognition}

Emotion recognition as a field dates back to Paul Eckman in the 70s [10]. It involves the recognition of different facial action units (FAUs) and the classification of those into basic emotions, typically seven. In [5], emotion detection is used to control a multirobot system in a bar service setting. An information robot detects the emotions of patrons. A server robot serves drinks in a one-to-one mapping of emotions to drinks and a music robot selects a track based on a one-to-one mapping of emotions to music. Based on the given system, emotions were correctly classified, and service robots selected the correct options. However, the satisfaction of customers was not evaluated, i.e. it was not determined whether the given framework was useful for the service scenario.

In some cases, it may be useful to determine actions based on emotion, such as for a social or therapeutic robot. In service tasks however, it may be more useful to use emotion as a reward function to determine if the task was completed satisfactorily. This would allow the robots to adapt their behavior based on the preferences of individual customers or clients. At the time of writing this paper, there is little research into this area, possibly due to the accuracy of current emotion recognition systems. Emotion can also be detected via speech, as in [22], or other physiological signals, as in [5]. These systems could be used in the same way as vision-based emotion detection but may be more applicable to specific environments or users.

\subsection{Gesture Based Robot Interfaces}

Gestures are a significant part of human interaction, and thus are another avenue to be explored for more natural HRI. Gestures can also act as another interface method to send commands to a robot. Gesture recognition is typically a computer vision problem; hands or arms are filtered out and the system determines shape or movement in order to classify a gesture as a command. Other methods include wearable devices, such as EMG armbands, accelerometers, or gyroscopes worn on the arm.

In [8], a framework for gesture-based robot control is introduced using wearable technology. Specifically, a smartwatch is worn on the right hand and is used to determine the movement of the hand. The designed interface was evaluated by subjects and found to be easy to learn and use; the gestures were intuitive and comfortable. In [16], head gestures are used in a robot interface. This study also uses wearable technology, with a nine-axis inertial measurement unit. Gesture recognition can also be solved with computer vision techniques. In [21], hand gestures are classified from video. Recurrent neural networks (RNNs) are combined with CNNs to increase the accuracy of gesture recognition. They achieve an accuracy of over $90 \%$. However, the combination of the two types of neural networks increases training time significantly.

In the future, along with evaluating gestures to use for different systems, different kinds of gestures could be combined. For example, arm and head gestures could be used to send different commands to robots, as they typically mean different things in human-human 
interaction. In robot control, this could allow for more commands while still using intuitive interfaces. In HRI this could allow robots to gather more information from a typical conversation. Additionally, improvements in training time for vision-based gesture recognition could further improve robot interfaces, as it would allow the use of gestures without requiring that the user wear additional devices.

\subsection{Lie Detection}

Another source of communication between humans is the ability to tell when someone is lying; this skill would be useful for robots as well. With lie detection, assistive robots could more effectively evaluate a user's mental and physical health. HRI would be more realistic and natural, and entertainment robots would have an additional area in which to expand.

Polygraph tests are the detection of physiological responses while a subject is asked a series of questions [15]. These responses typically include respiration, palmar sweat (i.e. sweating hands), and cardiovascular signals. There are different polygraph techniques, but they typically include asking the subject a series of questions in which some of the responses can be guessed and others are relevant to a case. To implement polygraph tests on a robot platform, a physiological response must be measured; a signal must be chosen that can be measured unobtrusively and accurately so as to not interfere with the rest of the interaction. One option is cardiovascular response, or pulse detection. [20] used thermal imaging to detect pulse, filter it, and determine truthfulness through use in a polygraph test. They achieved an accuracy of $84 \%$ on the test subjects. Pulse can also be detected via a standard camera. This is useful due to cost differences and the fact that many robots are designed with internal cameras already. [25] uses a standard camera to detect pulse. This method could be combined with polygraph methods to estimate truthfulness with only a camera.

\section{CONCLUSIONS}

Touchless and nonverbal interfaces are important for the advancement of robotics. They lead to further assistive technologies and more effective HRI. By creating intuitive and user friendly interfaces with touchless and nonverbal inputs, people with disabilities can interact with and benefit from assistive robots. Additionally, nonverbal communication will further the effectiveness of HRI, making it more likely that people will want to engage with the robot or system. The most common types of touchless and nonverbal interfaces are based on gaze detection, EEG or other biopotential signals, gestures, and emotion recognition.

Each method has its own limitations and areas for advancement. Gaze detection using near-infrared eye trackers is simple and accurate, but limits the situations that the system can be used. Computer vision may be less limited in gaze detection, but is currently less accurate and more dependent upon background variance. EEG is extremely accessible, but has a low SNR which can make it very hard to get accurate commands. Other biopotential signals can be limited by similar SNRs, device placement, and muscle fatigue. Gesture recognition often uses computer vision, which has the same dependencies on lighting and background as with eye tracking. Additionally, it can be challenging to assign commands or meaning to specific gestures in a way that is intuitive and easy for users.
Emotion recognition and lie detection are both potentially very useful for HRI, but both are complex topics which can be hard to define appropriate system responses even if the detection accuracy is high. If each of these fields is able to overcome these limitations, significant further advancements can be made with regards to touchless and nonverbal robot interfaces.

\section{REFERENCES}

[1] [n.d.]. Specification of Gaze Accuracy and Gaze Precision, Tobii X2-30 Eye Tracker. https://www.tobiipro.com/siteassets/tobii-pro/technical-specifications/ tobii-pro-x2-30-technical-specification.pdf/?v=1.0

[2] Amer Al-Rahayfeh and Miad Faezipour. 2014. Application of head flexion detection for enhancing eye gaze direction classification. 201436 th Annual International Conference of the IEEE Engineering in Medicine and Biology Society, EMBC 2014 (2014), 966-969. https://doi.org/10.1109/EMBC.2014.6943753

[3] P. Ballard and G. C. Stockman. 1992. Computer operation via face orientation. In [1992] Proceedings. 11th IAPR International Conference on Pattern Recognition. The Hague, Netherlands, 407-410.

[4] Christian J. Bell, Pradeep Shenoy, Rawichote Chalodhorn, and Rajesh P.N. Rao. 2008. Control of a humanoid robot by a noninvasive brain-computer interface in humans. Fournal of Neural Engineering 5, 2 (2008), 214-220. https://doi.org/10. 1088/1741-2560/5/2/012

[5] Luefeng Chen, Min Wu, Mengtian Zhou, Jinhua She, Fangyan Dong, and Kaoru Hirota. 2018. Information-Driven Multirobot Behavior Adaptation to Emotional Intention in Human-Robot Interaction. IEEE Transactions on Cognitive and Developmental Systems 10, 3 (2018), 647-658. https://doi.org/10.1109/TCDS.2017.2728003

[6] Anirban Chowdhury, Haider Raza, Ashish Dutta, and Girijesh Prasad. 2017. EEGEMG based hybrid brain computer interface for triggering hand exoskeleton for neuro-rehabilitation. ACM International Conference Proceeding Series Part F1320 (2017), 1-6. https://doi.org/10.1145/3132446.3134909

[7] Jeffrey F. Cohn and Edward Z. Tronick. 1987. Mother-Infant Face-to-Face Interaction: The Sequence of Dyadic States at 3, 6, and 9 Months. Developmental Psychology 23, 1 (1987), 68-77. https://doi.org/10.1037/0012-1649.23.1.68

[8] Enrique Coronado, Jessica Villalobos, Barbara Bruno, and Fulvio Mastrogiovanni. 2017. Gesture-based robot control: Design challenges and evaluation with humans. Proceedings - IEEE International Conference on Robotics and Automation 1 (2017), 2761-2767. https://doi.org/10.1109/ICRA.2017.7989321

[9] Trevor Lynn Craig, Carl A. Nelson, Songpo Li, and Xiaoli Zhang. 2016. Human gaze commands classification: A shape based approach to interfacing with robots. In 2016 12th IEEE/ASME International Conference on Mechatronic and Embedded Systems and Applications (MESA). IEEE, Auckland, 1-6. https://doi.org/10.1109/ MESA.2016.7587154

[10] Paul Ekman and Wallace V. Friesen. 1971. Constants across cultures in the face and emotion. Fournal of Personality and Social Psychology 17 (1971), 124-129. https://web-b-ebscohost-com.ezproxy.uta.edu/ehost/detail/detail?vid=8\{\&\}sid= b31892f2-afd0-4f1b-88c8-b95be726296b\{\%\}40pdc-v-sessmgr04\{\&\}bdata= JnNpdGU9ZWhvc3QtbGl2ZQ $\{\%\} 3 \mathrm{D}\{\%\} 3 \mathrm{D}\{\#\}$ AN $=1971-07999-001\{\&\} \mathrm{db}=$ pdh

[11] A. A. Fedorova, S. L. Shishkin, Y. O. Nuzhdin, and B. M. Velichkovsky. 2015. Gaze based robot control: The communicative approach. In 2015 7th International IEEE/EMBS Conference on Neural Engineering (NER). IEEE, Montpellier, 751-754. https://doi.org/10.1109/NER.2015.7146732

[12] David Feil-Seifer and Maja J. Matarić. 2005. Defining socially assistive robotics. In Proceedings of the 2005 IEEE 9th International Conference on Rehabilitation Robotics, Vol. 2005. 465-468. https://doi.org/10.1109/ICORR.2005.1501143

[13] Alan Fogel. 1977. Temporal organization in mother-infant face-to-face interaction. Studies in mother-infant interaction: proceedings of the Loch Lomond symposium January 1977 (1977).

[14] John Paulin Hansen, Alexandre Alapetite, I. Scott MacKenzie, and Emilie Møllenbach. 2014. The use of gaze to control drones. In Eye Tracking Research and Applications Symposium (ETRA). Safety Harbor, 27-34. https://doi.org/10.1145/ 2578153.2578156

[15] Charles R. Honts and Mary V. Perry. 1992. Polygraph admissibility. Law and human behavior 16, 3 (1992), 357-379. http://www.springerlink.com/index/ M367464MQ7818886.pdf\{\%\}5Cnpapers2://publication/uuid/C01BBBAA-002547C4-95CB-A043B28512B7

[16] Anja Jackowski, Marion Gebhard, and Axel Gräser. 2016. A novel head gesture based interface for hands-free control of a robot. 2016 IEEE International Symposium on Medical Measurements and Applications, MeMeA 2016 - Proceedings (2016). https://doi.org/10.1109/MeMeA.2016.7533744

[17] Jiaxin Ma, Yu Zhang, Andrzej Cichocki, and Fumitoshi Matsuno. 2015. A novel EOG/EEG hybrid human-machine interface adopting eye movements and ERPs: Application to robot control. IEEE Transactions on Biomedical Engineering 62, 3 (2015), 876-889. https://doi.org/10.1109/TBME.2014.2369483

[18] Ludovico Minati, Natsue Yoshimura, and Yasuharu Koike. 2016. Hybrid Control of a Vision-Guided Robot Arm by EOG, EMG, EEG Biosignals and Head Movement 
Acquired via a Consumer-Grade Wearable Device. IEEE Access 4 (2016), 95289541. https://doi.org/10.1109/ACCESS.2017.2647851

[19] Oskar Palinko, Alessandra Sciutti, Yujin Wakita, Yoshio Matsumoto, and Giulio Sandini. 2016. If looks could kill: Humanoid robots play a gaze-based social game with humans. IEEE-RAS International Conference on Humanoid Robots (2016), 905-910. https://doi.org/10.1109/HUMANOIDS.2016.7803380

[20] Ioannis Pavlidis and James Levine. 2002. Thermal Image Analysis for Polygraph Testing. IEEE Engineering in Medicine and Biology Magazine 21, 6 (2002).

[21] Lionel Pigou, Aäron van den Oord, Sander Dieleman, Mieke Van Herreweghe and Joni Dambre. 2018. Beyond Temporal Pooling: Recurrence and Temporal Convolutions for Gesture Recognition in Video. International fournal of Computer Vision 126, 2-4 (2018), 430-439. https://doi.org/10.1007/s11263-016-0957-7 arXiv: 1506.01911

[22] A. Revathy, P. Shanmugapriya, and V. Mohan. 2015. Performance comparison of speaker and emotion recognition. 2015 3rd International Conference on Signal Processing, Communication and Networking, ICSCN 2015 (2015), 1-6. https: //doi.org/10.1109/ICSCN.2015.7219844

[23] Margaret Gwendoline Riseborough. 1981. Physiographic gestures as decoding facilitators: Three experiments exploring a neglected facet of communication. Journal of Nonverbal Behavior 5, 3 (1981), 172-183. https://doi.org/10.1007/ BF00986134

[24] S. R. Soekadar, M. Witkowski, C. Gómez, E. Opisso, J. Medina, M. Cortese, M. Cempini, M. C. Carrozza, L. G. Cohen, N. Birbaumer, and N. Vitiello. 2016. Hybrid EEG/EOG-based brain/neural hand exoskeleton restores fully independent daily living activities after quadriplegia. Science Robotics 1, 1 (dec 2016), eaag3296. https://doi.org/10.1126/scirobotics.aag3296

[25] Michele Sorelli, Carlotta Kopietz, Sebastian Zaunseder, and Leonardo Bocchi. 2019. Pulse decomposition analysis in camera-based photoplethysmography.
Proceedings of the Annual International Conference of the IEEE Engineering in Medicine and Biology Society, EMBS (2019), 3179-3182. https://doi.org/10.1109/ EMBC.2019.8857856

[26] Piotr Stawicki, Felix Gembler, and Ivan Volosyak. 2016. Driving a Semiautonomous Mobile Robotic Car Controlled by an SSVEP-Based BCI. Computational Intelligence and Neuroscience 2016 (2016). https://doi.org/10.1155/2016/4909685

[27] Tian Xu, Hui Zhang, and Chen Yu. 2016. See you see me: The role of Eye contact in multimodal human-robot interaction. ACM Transactions on Interactive Intelligent Systems 6, 1 (2016), 1-22. https://doi.org/10.1145/2882970

[28] Bum Soo Yoo and Jong Hwan Kim. 2015. Fuzzy integral-based gaze control of a robotic head for human robot interaction. IEEE Transactions on Cybernetics 45, 9 (2015), 1769-1783. https://doi.org/10.1109/TCYB.2014.2360205

[29] Yasen Yordanov, Georgi Tsenov, and Valeri Mladenov. 2017. Humanoid robo control with EEG brainwaves. Proceedings of the 2017 IEEE 9th International Conference on Intelligent Data Acquisition and Advanced Computing Systems: Technology and Applications, IDAACS 20171 (2017), 238-242. https://doi.org/10. 1109/IDAACS.2017.8095083

[30] Guangtao Zhang and John Paulin Hansen. 2019. Accessible control of telepresence robots based on eye tracking. In Proceedings of the 11th ACM Symposium on Eye Tracking Research \& Applications (ETRA). Denver, 1-3. https://doi.org/10.1145/ 3314111.3322869

[31] Nannan Zhang, Jun Jiang, Jingsheng Tang, Zongtan Zhou, and Dewen Hu. 2014. A novel steady-state visually evoked potential-based brain-computer-interface paradigm to steer a humanoid robot. Proceedings - 2014 4th International Conference on Instrumentation and Measurement, Computer, Communication and Control, IMCCC 2014 (2014), 774-778. https://doi.org/10.1109/IMCCC.2014.164 